\title{
Metabolism of Acetoin
}

\section{THE FORMATION AND UTILIZATION OF ACETOIN AND BUTANE-2:3-DIOL IN THE DECEREBRATED CAT}

\author{
By J. DAWSON AND R. P. HULLIN \\ Department of Biochemistry, University of Leeds
}

(Received 7 May 1953)

In unpublished experiments on the blood levels of acetoin and butane-2:3-diol in cases of manicdepressive psychosis, we have noted variations in the total level of these compounds and in the ratio of their concentrations.

Previous workers (Neuberg \& Gottschalk, 1925; Greenberg, 1943) had demonstrated that injected acetoin was removed from the blood stream, only a small amount being excreted unchanged; no determinations of butane-2:3-diol were carried out. Westerfeld \& Berg (1943) also showed that injection of acetoin produced a small increase in acetoin excretion in the urine whilst the increase in urinary butane-2:3-diol excretion was much greater. The total excretion accounted for only some $12 \%$ of the amount injected. No increased formation of glycogen was observed and no suggestions offered as to the nature of the metabolic conversions occurring.

Doisy \& Westerfeld (1943) demonstrated that acetoin and butane-2:3-diol could act as acetyl donors for the acetylation of injected $p$-aminobenzoic acid, but the amount of acetylation attributable to these compounds would appear to be small and was a negligible part of the metabolic interconversions which acetoin and butane-2:3-diol undergo on injection. Although pyruvate is the most likely precursor of acetoin and butane-2:3-diol, Stotz, Westerfeld \& Berg (1944) were unable to demonstrate any increase in the blood level of these compounds after pyruvate injection. Acetaldehyde injection did, however, produce an increased level of acetoin and butane-2:3-diol in the blood.

The present investigation is concerned with the mechanism whereby blood levels of acetoin and butane-2:3-diol vary and also with the rate of disappearance of these compounds from the blood following injection. Since more sensitive methods were available for the estimation of these substances, smaller amounts could be injected and the time course of their removal followed.

\section{EXPERIMENTAL}

\section{Preparation of animals}

Cats of $2.5-4 \mathrm{~kg}$. body wt. were anaesthetized with ether, a cannula inserted in the trachea, and decerebrated after ligating the carotid arteries.
Cannulae were inserted in the carotid artery (to obtain blood samples) and in the femoral vein (to inject solutions) and the decerebrated cat was allowed to rest for $1 \mathrm{hr}$. Control values for the blood levels of acetoin, butane-2:3diol (BD), pyruvate and glucose were obtained from three blood samples taken at $30 \mathrm{~min}$. intervals after the rest period following decerebration. Each blood sample was treated with 2 vol. of $10 \%$ (w/v) trichloroacetic acid (TCA) and determinations of acetoin, $\mathrm{BD}$ and pyruvate made on the TCA filtrate. Extracellular fluid volume (ECF) was determined from the increase in serum thiocyanate after injection of $20 \mathrm{mg}$. of $\mathrm{NaCNS}$.

After the third blood sample had been collected, the test solution was injected slowly into the femoral vein, and further blood samples taken at $\mathbf{3 0} \mathrm{min}$. intervals. All solutions injected were adjusted to $\mathrm{pH}$ 7.0. An equimolar concentration of $\mathrm{NH}_{4} \mathrm{Cl}$ was injected with the sodium pyruvate solution in order to counteract any tendency towards alkalosis.

Urine samples were obtained immediately before each blood sample by intubation of the bladder. Two $3 \mathrm{ml}$. portions of $0.9 \% \mathrm{NaCl}$ were used to wash out the bladder each time.

\section{Methods}

Pyruvate was estimated on TCA filtrates of blood by the method of Friedemann \& Haugen (1943). Glucose determinations by the method of Hagedorn \& Jensen (see Hawk, Oser \& Summerson, 1947) were carried out on duplicate $0 \cdot 1 \mathrm{ml}$. samples of fresh blood.

Acetoin and BD were determined by the method of Happold \& Spencer (1952), slightly modified as follows for use on blood samples. The blood (about $5 \mathrm{ml}$.) was ejected from the syringe into a graduated centrifuge tube containing $10 \mathrm{ml}$. of cold $10 \%(\mathrm{w} / \mathrm{v}) \mathrm{TCA}$ and the mixture thoroughly shaken.

The accurate volume of the blood taken was measured after centrifuging for $20 \mathrm{~min}$. The supernatant solution was then decanted and the precipitated blood proteins reextracted with $12 \mathrm{ml}$. TCA. After further centrifuging, the supernatant solutions were combined and the volume of acid extract noted; preliminary experiments had shown that all the acetoin and $\mathrm{BD}$ were removed by the two extractions.

Acetoin was determined on a portion of the acid extract. The amount of acetoin and $\mathrm{BD}$ combined was determined on $6 \mathrm{ml}$. of the extract after adjusting to $\mathrm{pH} 7$ with $\mathrm{NaOH}$ solution, using bromothymol blue as external indicator. The precipitated hydrazone was washed with $5 \mathrm{ml}$. of $5 \%$ $(w / v)$ aqueous methanol to eliminate any possible interference from glucose. in the blood filtrate. Recovery experiments indicated that the method of Happold \& Spencer as modified for blood samples was accurate to within $\pm 5 \%$ 
and concentrations as low as $15 \mu \mathrm{g} . / 100 \mathrm{ml}$. of blood could be determined.

Thiocyanate was estimated by the method of Bowler (1944).

\section{RESULTS}

The effect of pyruvate injection on the blood concentration and total amount of acetoin and BD present in the ECF is given in Table 1. These results show that the blood pyruvate level can be raised by pyruvate injections and this rise is associated with an increased amount of acetoin distributed in the ECF but is not associated with any marked increase in the blood concentration. There is a significant rise in the blood level of $\mathrm{BD}$ following pyruvate injection but the total amount of $B D$ in the ECF shows a gradual rise throughout the experiment. It has been noted that in the decerebrated cat the blood glucose level gradually rises during the experiment irrespective of any injections made.

To clarify the effect of glucose on the blood concentration and the ECF content of acetoin and BD, massive glucose injections were made. The results are shown in Table 2. Neither the pyruvate level nor the ECF content is significantly increased by glucose injections which produced a marked rise in the blood concentration and total amount of glucose in the ECF. There is no significant change in blood or extracellular acetoin but the blood $\mathrm{BD}$ level and ECF content increase. Thus it is possible that the pyruvate injection increases the amount of acetoin in the ECF whilst glucose injection produces a rise in BD without apparently affecting the acetoin.

The injection of insulin (12 units/kg.) simultaneously with pyruvate had no effect on the acetoin rise and had no constant effect in limiting the increase of extracellular BD.

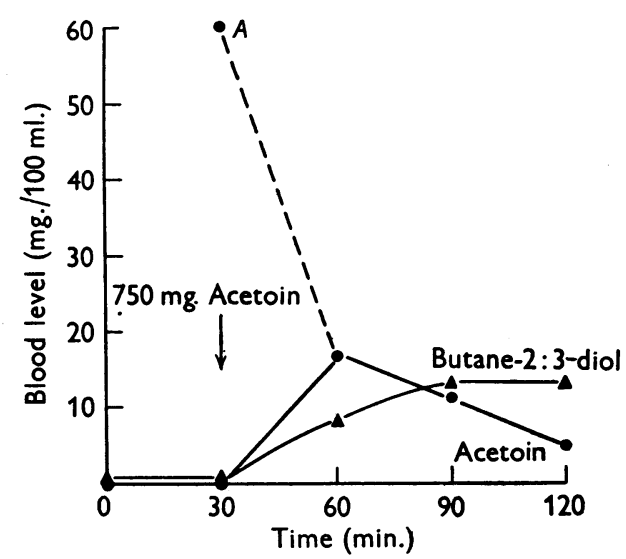

Fig. 1. The effect of an intravenous acetoin injection on the blood level of acetoin and butane-2:3-diol in the decerebrated cat. $A$ represents the blood level of acetoin at the instant of injection as calculated from the dose injected and the ECF volume of the preparation (1250 ml.).
Acetoin and BD were injected into decerebrated cats in order to investigate their subsequent metabolism. The results of representative experiments are shown in Figs. 1-3. The quantities of these compounds in the urine as well as in the blood were determined at $30 \mathrm{~min}$. intervals throughout the experiment. The amounts of both acetoin and BD in

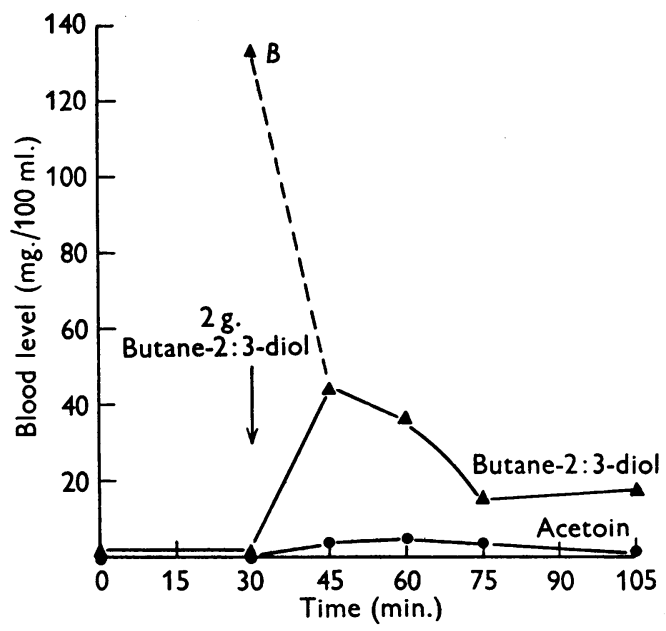

Fig. 2. The effect of an intravenous butane-2:3-diol (BD) injection on the blood level of acetoin and butane-2:3-diol in the decerebrated cat. $B$ represents the blood level of butane-2:3-diol at the moment of injection as calculated from the dose injected and the ECF volume of the preparation (1500 ml.).

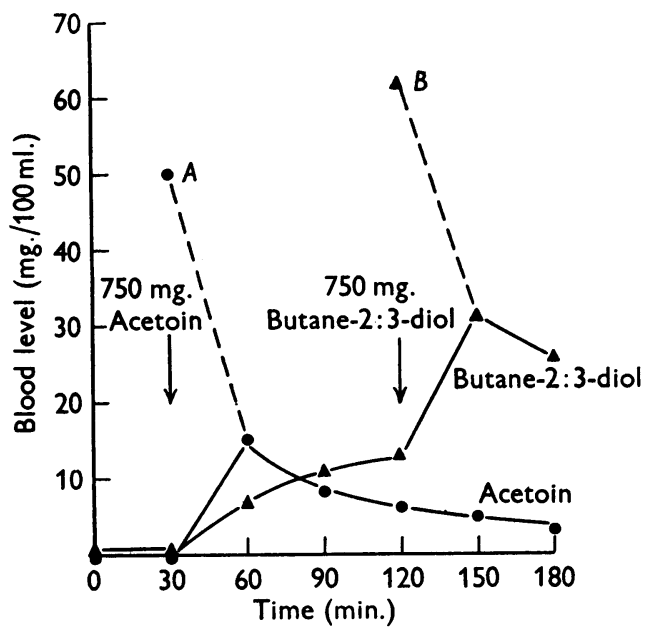

Fig. 3. The effect of successive intravenous injections of acetoin and butane-2:3-diol on the blood levels of these compounds in the decerebrated cat. $A$ represents the blood acetoin level at the moment of the first injection as calculated from the dose injected and the ECF volume of the cat, whilst $B$ indicates the corresponding butane-2:3diol value at the instant of the second injection. 


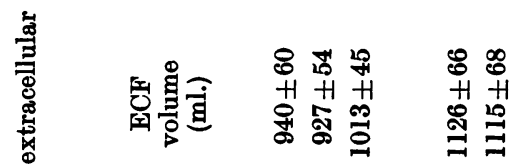

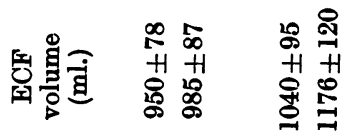

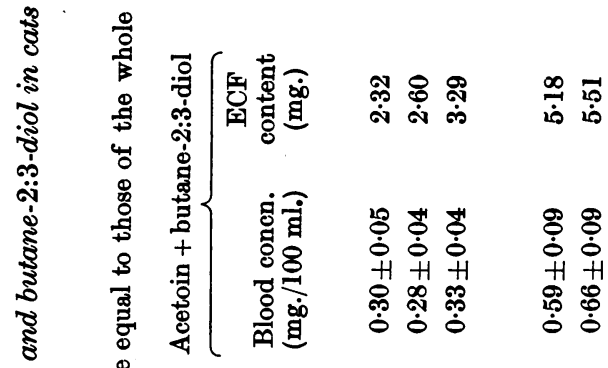

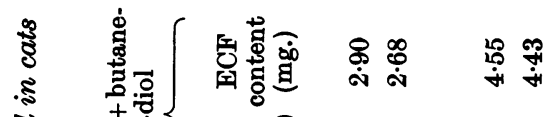

芯

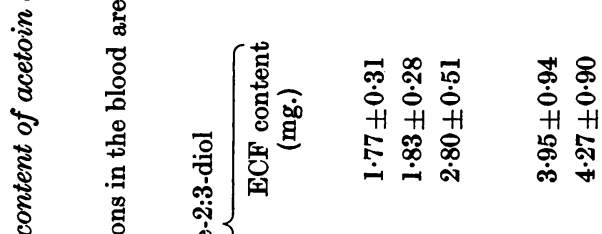

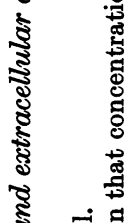

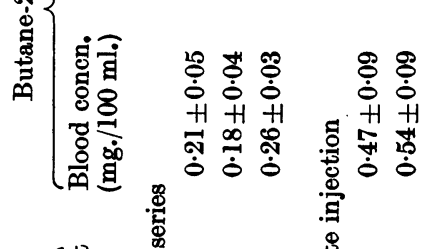

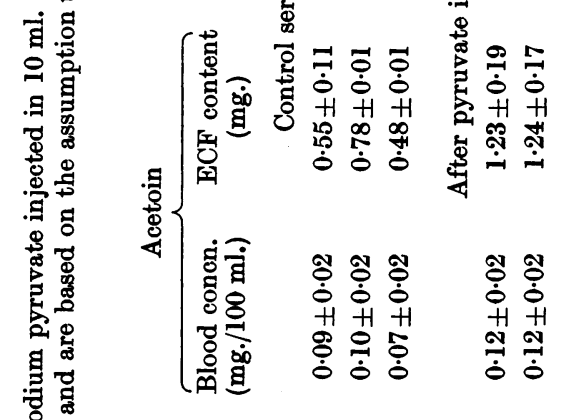

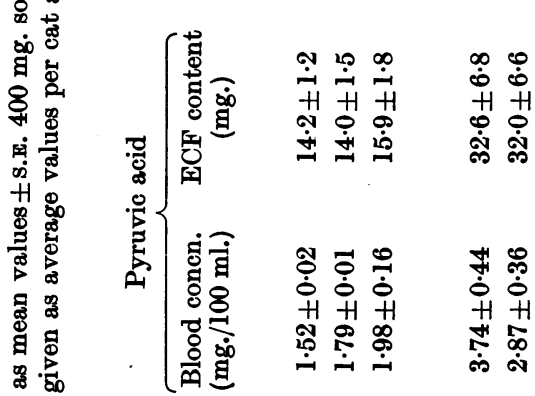

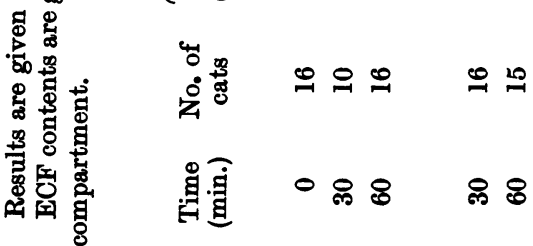

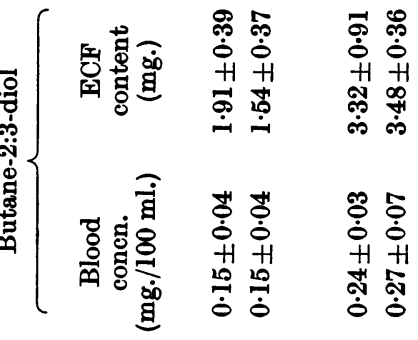

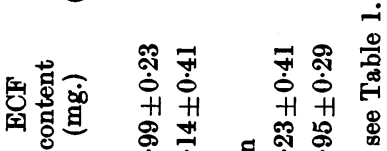

卷

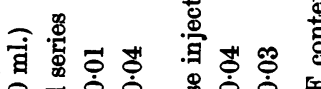

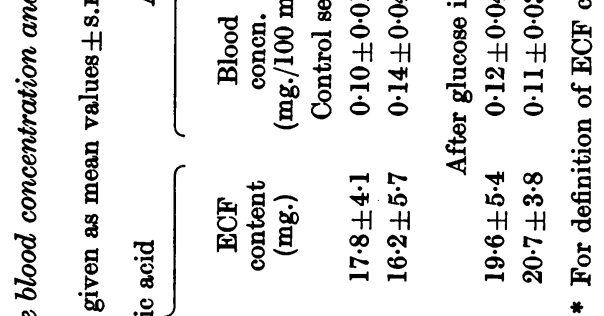

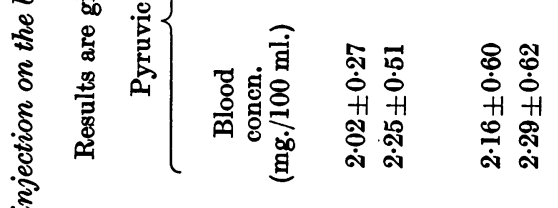

总

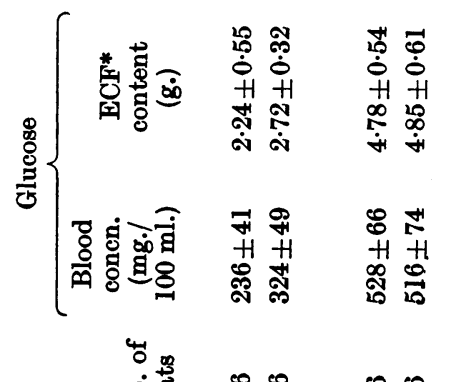

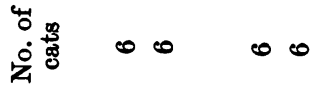

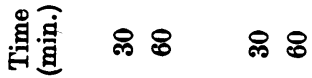


the urine increased after injection of either compound, the increase being mainly in the urinary BD. However, the total excretion of acetoin and $\mathrm{BD}$ in any urine sample never exceeded $0.1 \mathrm{mg}$., so that the rapid disappearance of these compounds from the circulation could not be attributed to excretion.

The results indicate that there is a rapid metabolism of both acetoin and BD. Acetoin injection produced a marked rise in the blood level of $B D$, while injection of BD caused only a minor rise in the blood acetoin concentration. At the end of each experiment the BD was still maintained at a level considerably above that present before injection, by an amount representing about $10 \%$ of the total of acetoin or BD injected. The decline from this latter level is slow.

\section{DISCUSSION}

When pyruvate is injected in doses large enough to produce a marked increase in blood pyruvate level, a small rise in acetoin production results. This could be due either to a small fraction of the metabolized pyruvate being converted into acetoin and butane-2:3-diol or to rapid metabolism of these compounds when produced. The proportion of pyruvate being converted in this way is unknown. Jagannathan \& Schweet (1952) reported that pyruvic oxidase in the absence of oxygen produces acetoin. In the intact animal, reduction of acetoin to butane-2:3-diol occurs in anoxaemia. Massive glucose injections do not influence acetoin production; this is not unexpected since the pyruvate level does not rise significantly. With the use of a more sensitive method for estimating acetoin and butane-2:3-diol it has been demonstrated that some pyruvate is converted into acetoin in the intact animal.

The metabolism of acetoin appears to be quite rapid and is associated with a considerable pro- duction of butane-2:3-diol. It is not known whether this is a step in the further metabolism of acetoin or whether it is a side reaction. From tissue dispersion experiments (Dawson \& Hullin, 1954) it appears that butane-2:3-diol can be metabolized as rapidly as acetoin. Injections of butane-2:3-diol do not produce a significant increase in acetoin level, however. This may indicate either that the normal route of butane-2:3-diol metabolism does not lie through acetoin, or that its rate of conversion to acetoin is slow compared with the further metabolism of the latter.

\section{SUMMARY}

1. The production and utilization of acetoin and butane-2:3-diol has been studied in decerebrated cats.

2. Pyruvate injection in such preparations increased acetoin production. Glucose injection had no such effect.

3. Injection of acetoin and butane-2:3-diol demonstrated that these compounds were rapidly metabolized.

\section{REFERENCES}

Bowler, R. G. (1944). Biochem. J. 38, 385.

Dawson, J. \&. Hullin, R. P. (1954). Biochem. J. 57, 180.

Doisy, E. A. \& Westerfeld, W. W. (1943). J.biol. Chem. 149, 229.

Friedemann, T. E. \& Haugen, G. E. (1943). J. biol. Chem. $147,415$.

Greenberg, L. A. (1943). J. biol. Chem. 147, 11.

Happold, F. C. \& Spencer, C. P. (1952). Biochim. biophys. Acta, 8, 18.

Hawk, P. B., Oser, B. L. \& Summerson, W. H. (1947). Practical Physiological Chemistry. London: Churchill.

Jagannathan, V.\& Schweet, R. S. (1952). J.biol. Chem.196, 551.

Neuberg, C. \& Gottschalk, A. (1925). Biochem. Z. 162, 484. Stotz, E., Westerfeld, W. W. \& Berg, R. L. (1944). J. biol. Chem. 152, 41.

Westerfeld,W.W.\& Berg, R.L. (1943).J.biol.Chem.148, 523.

\title{
Metabolism of Acetoin
}

\section{METABOLIC CONVERSIONS OF ACETOIN, PYRUVATE AND ACETATE BY RABBIT-KIDNEY TISSUE DISPERSIONS}

\author{
By J. DAWSON AND R. P. HULLIN \\ Department of Biochemistry, University of Leeds
}

(Received 7 May 1953)

In an unpublished study of the fasting blood levels of acetoin and butane-2:3-diol in normal subjects and in cases of manic-depressive psychosis, we have found that an increase in total acetoin plus butane2:3-diol level occurred in the depressed phases of the disorder while an increased butane-2:3-diol level associated with a decreased acetoin level was observed during the manic phases. These changes could have been due either to an increased production or to a decreased catabolism of these fourcarbon compounds.

From experiments with decerebrated cats, it has also been shown (Dawson \& Hullin, 1954) that the acetoin content of the extracellular fluid can be 